\title{
Clinical Trial Subject Screen Log
}

National Cancer Institute

\section{Source}

National Cancer Institute. Clinical Trial Subject Screen Log. NCI Thesaurus. Code

C115538.

Records of all subjects considered for participation in a clinical trial. 